\title{
Method to Improve Propeller Performance Using Real-coded Genetic Algorithm*
}

\author{
Jun Ando** Shiro Kataoka*** Tomohiro Ryu***
}

\begin{abstract}
This paper presents a numerical optimization method to improve propeller performance using real-coded genetic algorithm. In the method presented, UNDX (Unimodal Normal Distribution Crossover) and MGG (Minimal Generation Gap) models are used as crossover operator and generation-alternation models, respectively. Propeller performance is evaluated by a simple surface panel method "SQCM" in the optimization process. The blade section of the original propeller is replaced by the NACA66 $\mathrm{a}=0.8$ section. However, original chord, skew, rake and maximum blade thickness distributions in the radial direction are unchanged. Pitch and maximum camber distributions in the radial direction and the position of maximum camber in the chord wise direction are selected as the design variables. Optimization is conducted to maximize the propeller efficiency while keeping the target thrust coefficient constant. Cavitation performance is also considered as a constraint condition. Propellers for a product tanker and a cargo ship were improved by utilizing the present method. Propeller open tests and cavitation tests of the original and improved propellers were carried out. It was confirmed that both improved propellers had higher efficiencies compared to those of the original propellers. It was also found that both cavitation performances of the improved propellers are permissible.
\end{abstract}

\section{INTRODUCTION}

Recently, energy saving in ship propulsion is increasingly important for the request of the $\mathrm{CO} 2$ emissions reduction leading to global warming, and the fuel consumption reduction by oil price jump. Since propeller performance has great influence on the propulsive performance of a ship, propeller design is an important technology for energy

\footnotetext{
* $\quad$ Received February16,2011

** Kyushu University, Fukuoka, Japan

*** Shin Kurushima Dockyard Co., LTD., Imabari, Ehime, Japan
}

saving in ship propulsion.

As for the method of propeller design, the traditional method using the propeller design chart obtained from the systematic model experiments is still common. Lifting surface method and panel method are also used for propeller design in a trial and error manner. Moreover, propeller design methods using optimizing techniques have been presented. Kawakita and Hoshino ${ }^{1)}$ designed the new blade sections with the prescribed pressure distributions on the propeller blade by the SUMT(Sequential Unconstrained Minimization Technique)method. Takekoshi et al. ${ }^{2)}$ used the SQP (Sequential Quadratic Programming) method to maximize the propeller efficiency under complex restrictions. Karim et $a l^{3)}$ and Jung et $a l^{4)}$ presented optimization techniques using genetic algorithm.

In this paper, a numerical optimization method to improve propeller performance using real-coded genetic algorithm is proposed. Propellers for a product tanker and a cargo ship are improved by the present method. Outline of the present method is described and model experiments results are shown.

\section{NUMERICAL OPTIMIZATION METHOD TO IMPROVE PROPELLER PERFORMANCE}

\subsection{Concept of Improvement Method}

In the present method, original chord, skew, rake and maximum blade thickness distributions in the radial direction are unchanged. Many different propellers are generated by changing the pitch and maximum camber distributions in the radial direction. Blade sections are also changed. The hydrodynamic characteristics of the propellers are calculated in a short time by the panel method described later. Then these calculated results are processed by an optimization technique. Finally an improved propeller can be obtained automatically. Thus, the present method does not create a new propeller at all from the state of nothing. Therefore, it is defined as a support tool for propeller design using an optimization technique. 
In order to calculate the hydrodynamic characteristics of propellers, a simple surface panel method "SQCM"5), 6) which was developed at Kyushu University is used in the present method. SQCM can satisfy the Kutta condition with no iterations even in 3-D problems. Since the computational time is short, SQCM is suitable for a numerical optimization method. As for the numerical optimization method, the real-coded genetic algorithm using $\mathrm{UNDX}^{7), 8)}$ and $\mathrm{MGG}^{9)}$ as crossover operator and generation-alternation model is adopted. Optimization is conducted to maximize the propeller efficiency keeping the target thrust. Cavity area on the propeller blade is also considered as a constraint condition in a simple way.

\subsection{Real-coded Genetic Algorithm}

The genetic algorithm (GA) is a stochastic optimization technique inspired by the evolution process of natural life. In GA, selection is performed in the population of a certain generation so that an individual with high fitness to the objective function in the optimization problem survives with high probability. Furthermore, the population of the next generation is formed by crossover and mutation. As alternation of generations proceeds, the individuals with higher fitness increase, and the most suitable solution is provided. The above is a basic concept of GA.

In general, an individual is expressed by binary string of 0 or 1 of the suitable number per one design variable in GA. And this binary string is transformed to the design variable which is a real number. The chromosome of each individual is expressed by binary strings of the same number as the number of the design variables. Spaces expressed by binary strings and real numbers are called genotype and phenotype spaces, respectively. The mapping from phenotype to genotype is called coding. The GA with coding by binary string is called binarycoded GA. And binary-coded GA is applied to various problems. On the other hand, several GAs which use the real number directly to express an individual have been proposed. This kind of GA is called real-coded GA.

It has been reported that real-coded GA can surely find the optimum solution if the design variables are continuous in function optimization problems ${ }^{7}$. In the present study, the real-coded GA using UNDX (Unimodal Normal Distribution Crossover) as a crossover operator is adopted. This GA was applied to the lens design problem, which is known as a difficult problem, and its usefulness was confirmed $^{8)}$. UNDX proposed by Ono et al. ${ }^{7)}$ is a kind of crossover operator in real-coded GAs. Each individual is defined by a real number vector and the dimension of the vector space is the same number as the number of design variables $n_{D}$. Two offspring vectors $\vec{C}_{1}, \vec{C}_{2}$ are generated by the normal distribution which is defined by three parents $\vec{P}_{1}, \vec{P}_{2}$ and $\vec{P}_{3}$, as shown in Figure 1 . One of the standard deviation values of the normal distribution, which corresponds to the principal axis connecting Parent 1 and Parent 2, is proportional to the distance between Parent 1 and Parent 2. The other is proportional to the distance of the third parent, Parent 3, from the principal axis connecting Parent 1 and Parent 2 and multiplied by $1 / \sqrt{n_{D}}$.

Offspring vectors are expressed by the following equations.

$$
\begin{aligned}
& \vec{C}_{1}=\vec{M}+z_{1} \vec{e}_{1}+\sum_{k=2}^{n_{D}} z_{k} \vec{e}_{k} \\
& \vec{C}_{2}=\vec{M}-z_{1} \vec{e}_{1}-\sum_{k=2}^{n_{D}} z_{k} \vec{e}_{k}
\end{aligned}
$$

Where

$$
\begin{aligned}
& \overrightarrow{\boldsymbol{M}}=\left(\overrightarrow{\boldsymbol{P}}_{1}+\overrightarrow{\boldsymbol{P}}_{2}\right) / 2 \\
& z_{1} \sim N\left(0, \sigma_{1}^{2}\right), \quad z_{k} \sim N\left(0, \sigma_{2}^{2}\right),\left(k=2, \cdots, n_{D}\right) \\
& \sigma_{1}=\alpha d_{1}, \quad \sigma_{2}=\beta d_{2} / \sqrt{n_{D}} \\
& \vec{e}_{1}=\left(\overrightarrow{\boldsymbol{P}}_{1}-\overrightarrow{\boldsymbol{P}}_{2}\right) /\left|\overrightarrow{\boldsymbol{P}}_{1}-\overrightarrow{\boldsymbol{P}}_{2}\right| \\
& \vec{e}_{i} \perp \vec{e}_{j}, \quad(i \neq j), \quad\left(i, j=1, \cdots, n_{D}\right)
\end{aligned}
$$

Here $z_{1}$ and $z_{k}$ are normal random numbers. $\sigma_{1}$ and $\sigma_{2}$ are standard deviations. $\boldsymbol{d}_{1}$ is the distance between Parent 1 and Parent $2, \boldsymbol{d}_{2}$ is the distance of the third parent, Parent 3 , from the principal axis connecting Parent 1 and Parent 2. $\alpha$ and $\beta$ are constants defined by parameter study and $\alpha=0.5$ and $\beta$ $=0.35$ are recommended ${ }^{7}$. Vectors $\vec{e}_{i}\left(i=2, \cdots, n_{D}\right)$ are the orthogonal basis vectors spanning the subspace perpendicular to vector $\vec{e}_{1}$. 


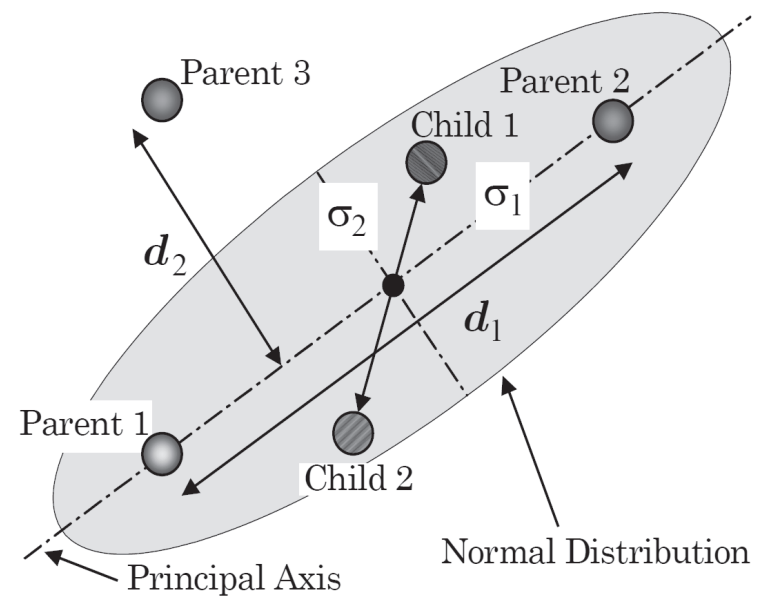

Figure 1 Concept of UNDX

In GA, it is very important to use a generation-alternation model which can avoid the early convergence and suppress the evolutionary stagnation. In the present study, MGG (Minimal Generation Gap) model proposed by Satoh et al. ${ }^{9 \text { ) }}$ is adopted. The general algorithm of the real-coded GA used in the present study is described as follows (see Figure 2):

\section{(Step 1) Generation of initial population}

Make an initial population of size $\boldsymbol{N}_{P}$ by generating the design variables randomly.

\section{(Step 2) Selection for reproduction}

Select a pair of parents from the current population randomly.

\section{(Step 3) Generation of offsprings}

Generate $2 \times N C$ offsprings by applying UNDX to the selected pair of parents $N_{C}$ times. The third parent which is used for calculating the standard deviation value, $\sigma_{2}$, is randomly chosen from the population.

\section{(Step 4) Selection for survival}

Evaluate the objective functions for the pair of parents selected at Step 2 and $2 \times N C$ offsprings generated at Step 3. Select two individuals among them; one is the best individual and the other is selected from $2 \times N_{C}+1$ individuals other than the best one by the rank-based roulette wheel selection. Replace the parents selected at Step $\mathbf{2}$ in the population with the two individuals.

Repeat the above procedures from Step 2 to Step 4 until a certain condition is satisfied.

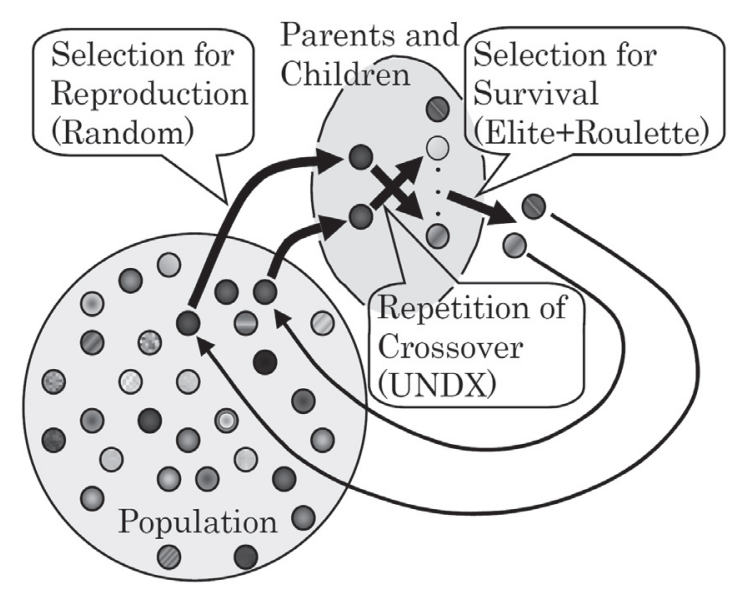

Figure 2 MGG model

\subsection{Definition of Optimization Problem}

\subsubsection{Design Variables}

In the present method, the improved propeller has the same chord, skew, rake and maximum blade thickness distributions in the radial direction as the original propeller. The number of the blade is also the same. Thickness and camber distributions in the chord-wise direction may be used the same ones as the original propeller or other distributions may be adopted. The blade section used for the improved propeller is called basic blade section.

Pitch and maximum camber distributions in the radial direction are selected as the design variables. These shapes are approximated by parabolic functions. The position of the maximum cambers in the chord-wise direction may be changed too.

\subsubsection{Objective Function}

Optimization is conducted to maximize the propeller efficiency $\eta_{\mathrm{O}}$ keeping the target thrust coefficient $\boldsymbol{K}_{T}^{\text {Target }}$

The objective function $\boldsymbol{F}$ is expressed by the following equation with the penalty function $\boldsymbol{P}$.

$$
\boldsymbol{F}=\boldsymbol{P} \times \eta_{0}
$$

Where

$$
\boldsymbol{P}=\exp \left(-\left|\boldsymbol{K}_{T}-\boldsymbol{K}_{T}^{\text {Target }}\right| / \boldsymbol{K}_{T}^{\text {Target }}\right)
$$

Here the maximization problem expressed by Equation (2) is solved by SQCM.

\subsubsection{Constraint Condition}

As the constraint condition for cavitation performance, the amplitude of fluctuating pressure at the 1st blade frequency 
above propeller center calculated by Holden'smethod ${ }^{10)}$ is considered. First the fluctuating pressure amplitude of the original propeller at the 1st blade frequency is calculated and determined the upper limit of the fluctuating pressure amplitude. If the cavity area is required not to exceed the original one, equivalent value to the original amplitude of fluctuating pressure at the 1stblade frequency is imposed as the upper limit.

\subsubsection{Procedure of Optimization}

The procedure of optimization for improving propeller performance is described as follows:

\section{(Step 1) Generation of initial population}

Obtain the pitch and maximum camber distributions in the radial direction by generating the design variables randomly. Make the propeller geometric data by considering chord, skew, rake and maximum blade thickness distributions in the radial direction of the original propeller and thickness and camber distributions in the chord-wise direction of the basic blade section.

Calculate the propeller performance by SQCM. Calculate the amplitude of fluctuating pressure at the 1st blade frequency by Holden's method. If the calculated fluctuating pressure amplitude is lower than the imposed upper limit, the propeller is permitted to join the initial population.

Repeat the above procedure until the number of the individuals (propellers) which satisfy the constraint condition for the pressure fluctuation becomes $N P$.

\section{(Step 2) Selection for reproduction}

Select a pair of parents from the current population randomly.

\section{(Step 3) Generation of offsprings}

Generate two offsprings (propellers) by applying UNDX to the selected pair of parents at Step 2. Calculate the propeller performance and the fluctuating pressure amplitude by SQCM and Holden's method, respectively. If the calculated fluctuating pressure amplitude is lower than the imposed upper limit, the propeller is selected as an object of evaluation at the next step. Calculate the objective function by Equation (2) and memorize the calculated value.

Repeat the above procedure until the number of the offsprings which satisfy the constraint condition for the pressure fluctuation becomes $2 \times \mathrm{NC}$.

\section{(Step 4) Selection for survival}

Select two individuals from the pair of parents selected at
Step 2 and $2 \times N C$ offsprings generated at Step 3; one is the best individual and the other is selected from $2 \times N_{C}+1$ individuals other than the best one by the rank-based roulette wheel selection. Replace the parents selected at Step $\mathbf{2}$ in the population with the two individuals.

Repeat the above procedure from Step 2 to Step 4 until a certain condition is satisfied.

\section{MODEL EXPERIMENTS RESULTS}

Propellers for a product tanker and a cargo ship were improved by the present method. The NACA66 section with $\mathrm{a}=0.8$ mean camber line was used for the improved propellers as the basic blade section. Both propellers were improved under full load condition. Propeller open tests and cavitation tests for original and improved propellers were conducted at Shipbulding Research Centre of Japan.

\subsection{Propeller for a product tanker}

Principal particular of the original propeller is shown in Table 1 . The design point $\boldsymbol{K} \boldsymbol{T} / \boldsymbol{J}^{2}$ was 0.800 . Cavity area of the improved propeller was allowed to be the same extent as the original propeller. So the upper limit of the amplitude of fluctuating pressure at the 1st blade frequency calculated by the Holden's method was set tot he same value as the original one.

Table 1 Principal particular of original propeller for product tanker

\begin{tabular}{|l|c|}
\hline Blade section & MAU \\
\hline Diameter (m) & 6.0 \\
\hline Number of blade & 4 \\
\hline Pitch ratio (0.7R) & 0.653 \\
\hline Expanded area ratio & 0.515 \\
\hline Boss ratio & 0.160 \\
\hline Rake angle (deg.) & 4.0 \\
\hline Skew angle (deg.) & 30.0 \\
\hline
\end{tabular}

The pitch distributions in the radial direction are shown in Figure 3.

The results of propeller open tests are shown in Figure 4. The efficiency of the improved propeller is $1.97 \%$ higher than that of the original propeller at the design point. 


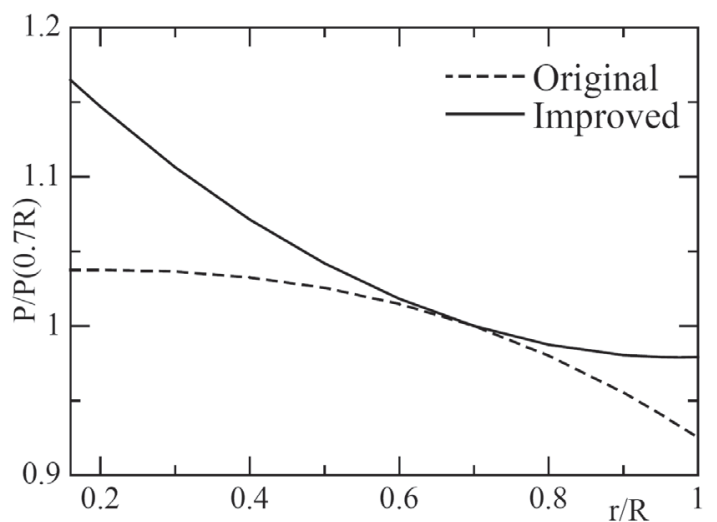

Figure 3 Pitch distributions

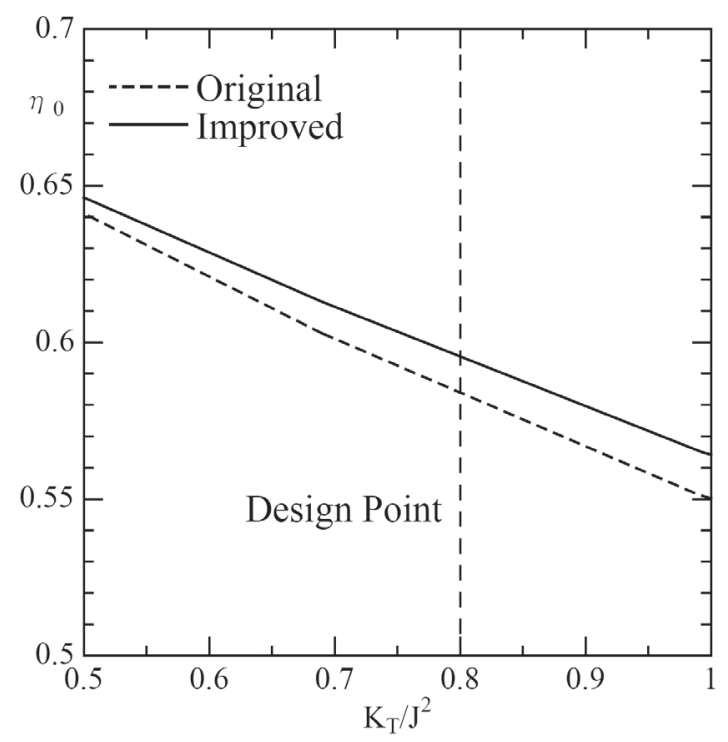

Figure 4 Comparison of propeller efficiency

The observed cavitation patterns of the original and improved propellers are shown in Figure 5. In the cavitation test, the wake was simulated by wire mesh screen. Cavitation number $\sigma_{\mathrm{N}}$ was 1.74 . The cavity area of the improved propeller is smaller than that of the original one.

Measured pressure fluctuations of 1 st and 2nd blade frequency above propeller center are shown in Figure 6.All values in the figure are normalized by the amplitude of the original propeller at the 1st blade frequency. The pressure fluctuation levels of the improved propeller at the 1st and 2nd blade frequency are about $40 \%$ and $20 \%$ lower than those of the original propeller, respectively.

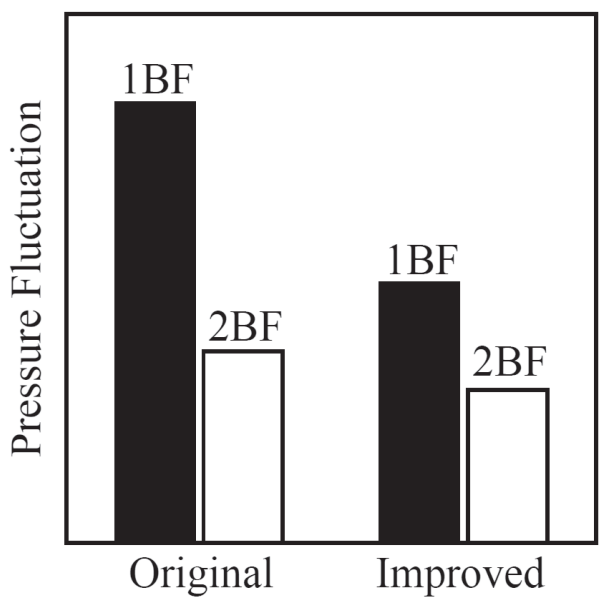

Figure 6 Comparison of pressure fluctuations of 1 st and 2nd blade frequency

\subsection{Propeller for a cargo ship}

Principal particular of the original propeller is shown in Table 2. The design point $\boldsymbol{K}_{\boldsymbol{T}} / \boldsymbol{J}^{2}$ was 0.882 . As for this propeller, the constraint condition for the cavity area was mitigated in order to improve the propeller efficiency primarily. Then $13 \%$ higher amplitude of fluctuating pressure of the original propeller at the 1st blade frequency was given as the upper limit.

Table 2 Principal particular of original propeller

\begin{tabular}{|l|c|}
\multicolumn{1}{c}{ for cargo ship } \\
\hline Blade section & Modified KIS \\
\hline Diameter (m) & 5.15 \\
\hline Number of blade & 5 \\
\hline Pitch ratio (0.7R) & 0.729 \\
\hline Expanded area ratio & 0.585 \\
\hline Boss ratio & 0.186 \\
\hline Rake angle (deg.) & 2.0 \\
\hline Skew angle (deg.) & 40.0 \\
\hline
\end{tabular}

The pitch distributions in the radial direction are shown in Figure 7.The results of propeller open tests are shown in Figure 8 . The efficiency of the improved propeller is $1.82 \%$ higher than that of the original propeller at the design point. 


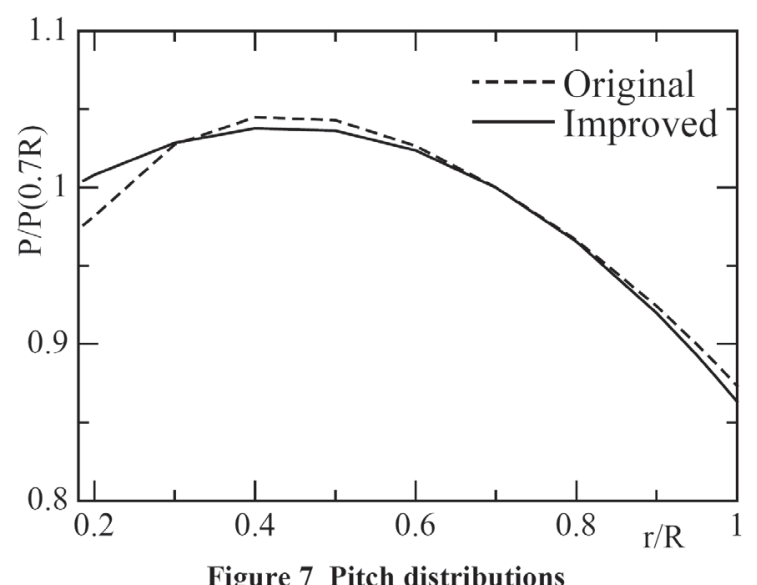

Figure 7 Pitch distributions

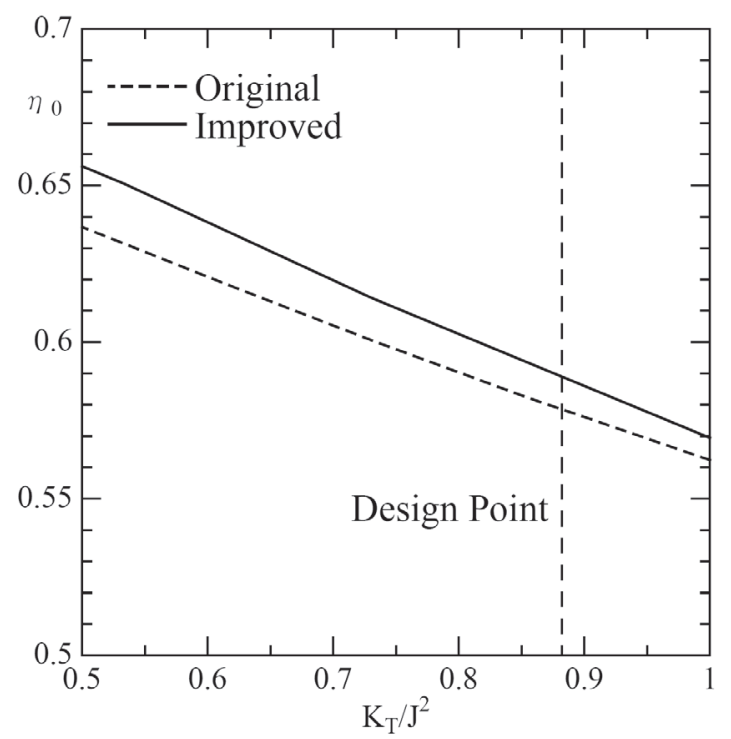

Figure 8 Comparison of propeller efficiency

The observed cavitation patterns of the original and improved propellers are shown in Figure 9. Cavitation number $\sigma_{\mathrm{N}}$ was 2.04. Though the cavity area of the improved propeller increased slightly, the cavity area of the improved propeller is within permissible range.

\section{CONCLUSIONS}

A Method to improve the propeller performance using real-coded genetic algorithm was developed. In order to investigate the usefulness of the present method, propellers for a product tanker and a cargo ship were improved and propeller open tests and cavitation tests of the original and improved propellers were carried out. It was confirmed that both improved propellers had higher efficiencies compared to the original propellers. It was also found that both cavitation performances of the improved propellers were permissible. The present method is a useful support tool for propeller design.

\section{REFERENCES}

1) C. Kawakita and T. Hoshino: Design system of marine propellers with new blade sections, Proceedings of the $22^{\text {nd }}$ Symposium on Naval Hydrodynamics, 1998, pp.110-126.

2) Y. Takekoshi et.al. : Study on the design of propeller blade sections using the optimization algorithm, Journal of Marine Science and Technology, Vol. 10, No. 2, 2005, pp.70-81.

3) M. M. Karim et.al. : Application of micro-genetic algorithm( $\mu$ GA) to the optimal design of lifting bodies, Journal of the Kansai Society of Naval Architects, Japan, No. 235, 2001, pp.1-8.

4) J. Jung et.al. : Design of marine propellers using genetic algorithm, Proceedings of the 10th International Symposium on Practical Design of Ships and Other Floating Structures, 2007, pp.1193-1199.

5) J. Ando et.al. : A simple surface panel method to predict steady marine propeller performance, Journal of the Society of Naval Architects of Japan, Vol. 178, 1995, pp.61-69.

6) J. Ando et.al. : A new surface panel method to predict steady and unsteady characteristics of marine propeller, Proceedings of the 22nd Symposium on Naval Hydrodynamics, 1998, pp.142-154.

7) I. Ono et.al. : A real-coded genetic algorithm for function optimization using the unimodal normal distribution crossover, Journal of Japanese Society for Artificial Intelligence, Vol. 14, No. 6, 1999, pp.1146-1155.

8) I. Ono et.al. : Optimal lens design by real-coded genetic algorithms using UNDX, Computer Methods in Applied Mechanics and Engineering, Vol. 186, 2000, pp.483-497.

9) H. Satoh et.al. : A new generation alternation method of genetic algorithms and its assessment, Journal of Japanese Society for Artificial Intelligence, Vol. 12, No. 5, 1997, pp.734-744.

10) Holden, K.O.: Excitation forces and afterbody vibrations induced by marine propeller blade cavitation, Norwegian Maritime Research, Vol. 7, No. 1., 1979, pp.15-26. 

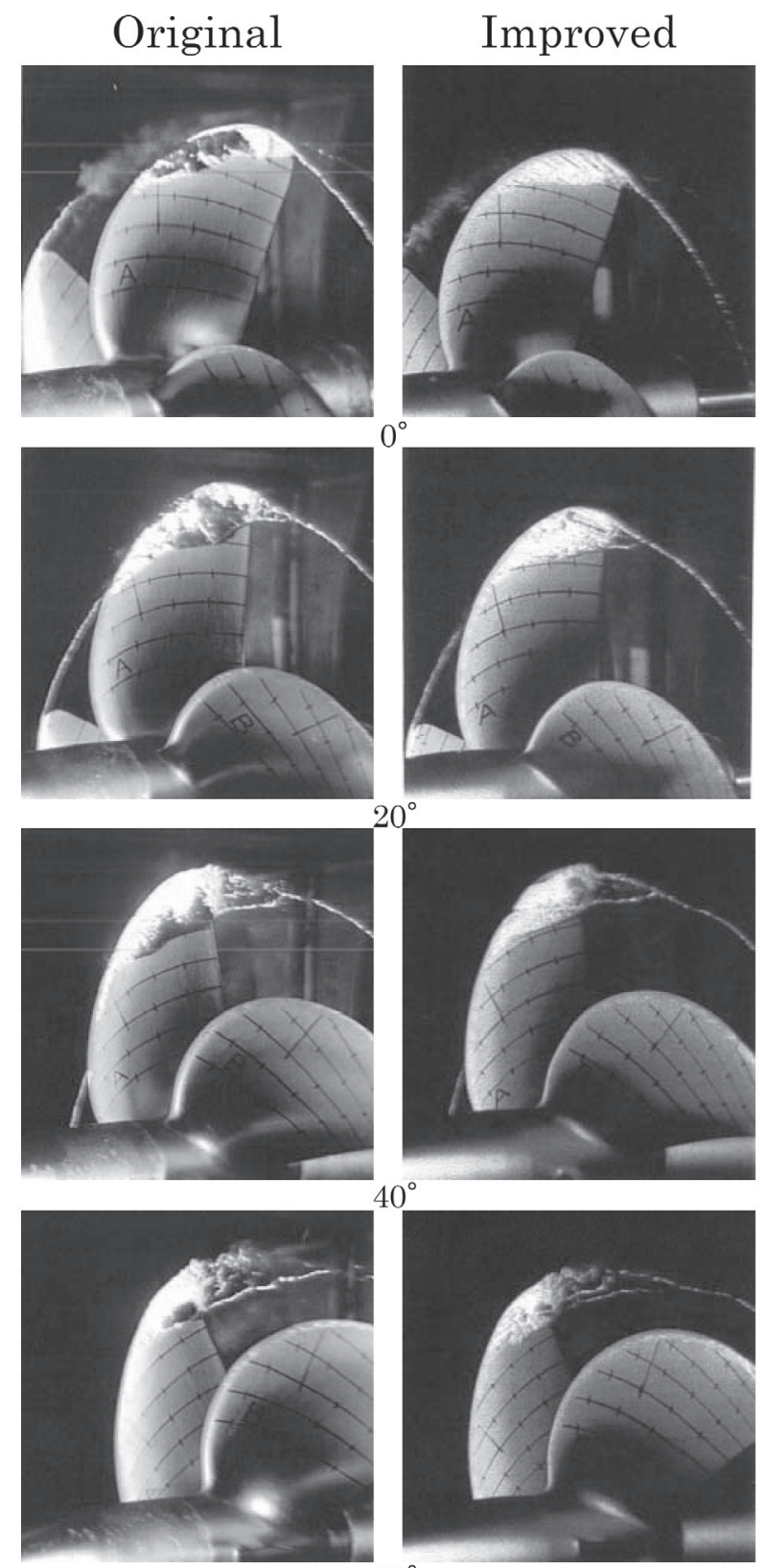

$60^{\circ}$

Figure 5 Cavitation patterns of original and improved propellers for product tanker

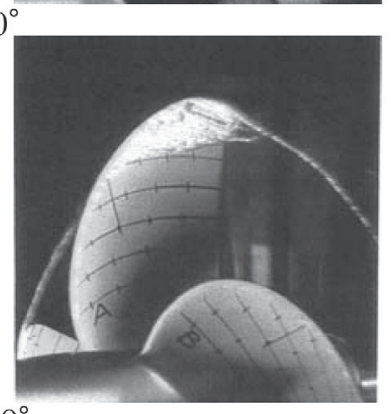

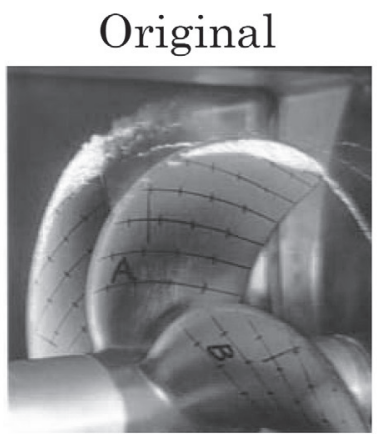
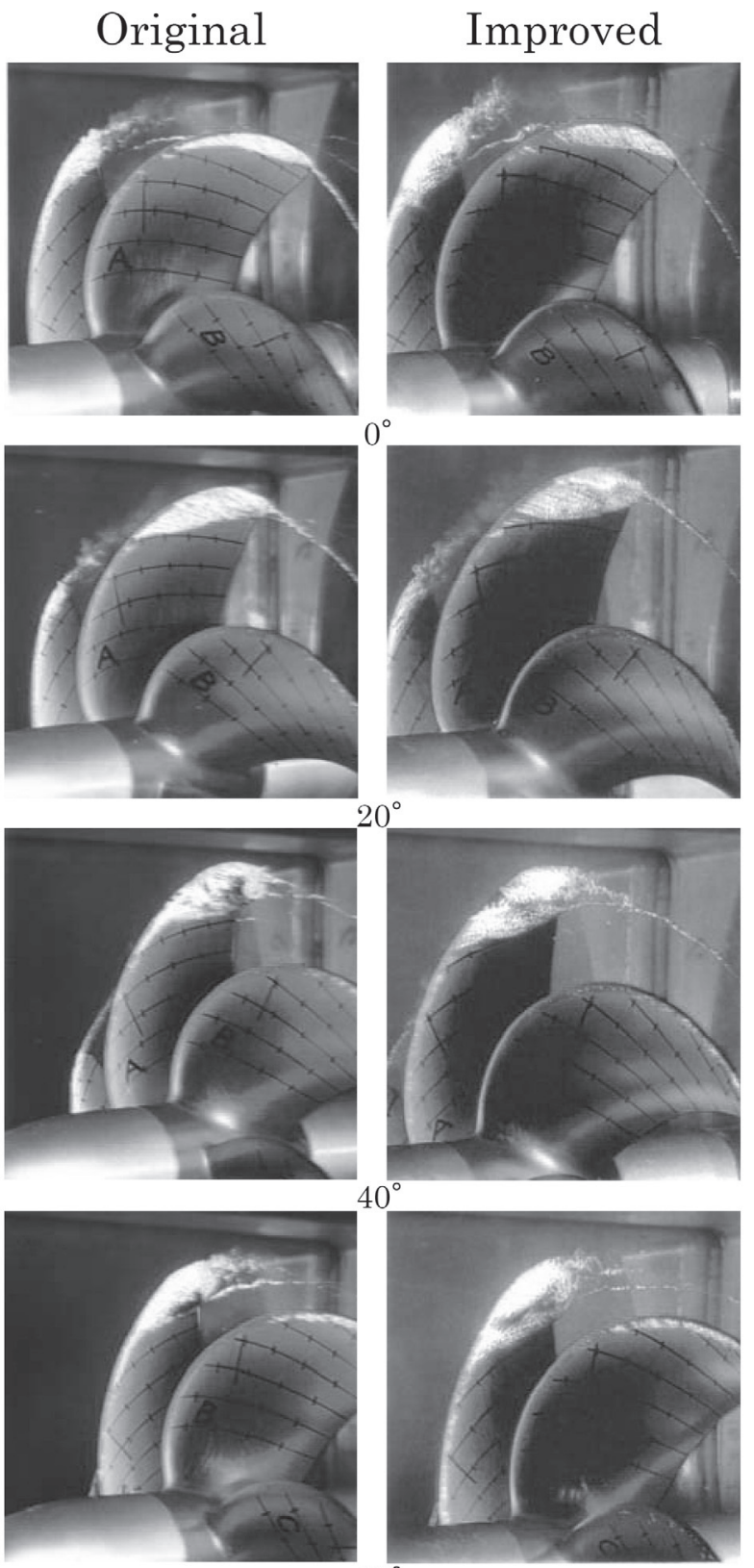

$60^{\circ}$

Figure 9 Cavitation patterns of original and improved propellers for cargo ship 